\title{
The Impact of Minimally Invasive Treatment for Rotator Cuff Calcific Tendinitis on Self-Reported Work Ability and Sick Leave
}

\author{
Jan K. G. Louwerens, M.D., P. Paul F. M. Kuijer, Ph.D., Inger N. Sierevelt, M.Sc., \\ Michel P. J. van den Bekerom, M.D., Ph.D., Barend J. van Royen, M.D., Ph.D., \\ Denise Eygendaal, M.D., Ph.D., and Arthur van Noort, M.D., Ph.D.
}

\begin{abstract}
Purpose: To examine the impact of rotator cuff calcific tendinitis on patients' self-reported work ability and sick leave, to compare work ability and sick leave with shoulder function after minimally invasive treatment, and to assess which prognostic factors influence the change in work ability. Methods: A prospective cohort was analyzed in this study. The primary outcome measure was the single-question work ability score (0-10 points). Secondary outcome measures were quality and quantity of work, sick leave, functional outcome, and radiographic resorption. Potential predictive factors (treatment method, age, sex, resorption of the calcific deposit, physical work load, and work status) were tested in a statistical model. Follow-up was at 6 months and 1 year. Results: The study cohort consisted of 67 patients. The mean age was $49.6 \pm 6.4$ years and $45(67 \%)$ were female. Physical workload was categorized as light $(58 \%)$, medium $(24 \%)$, and heavy $(18 \%)$. Work ability score improved from a mean of $6.1 \pm 2.8$ to $8.5 \pm 2.0$ points after 1 year. Treatment with minimally invasive treatment techniques was associated with a reduction in partial or full-time sick leave from $28 \%$ to $6 \%$. The mean days of sick leave a month declined from 3.3 to 0.8 days. Functional disability was greater in patients with partial or full-time sick leave. The physical workload turned out to be the most important patient associated factor predicting change in work ability. Conclusions: This study supports the hypothesis that rotator cuff calcific tendinitis has a significant impact on work ability and sick leave. Minimally invasive treatment resulted in a clinically relevant improvement in work ability score and decline in sick leave. In particular, patients with medium and high physically demanding work for the shoulder benefit from minimally invasive treatment to improve their work ability. Level of Evidence: Level II, prospective comparative study.
\end{abstract}

$\mathbf{S}^{\mathrm{h}}$ houlder problems are common in the Netherlands, with an incidence in the primary care of around 19 per 1000 person-years. ${ }^{1}$ Klik of tik om tekst in te voeren. Shoulder disorders represent various clinical diagnoses, varying from International Classification of Diseases codes M75.0 to M75.5. Rotator cuff calcific tendinitis (RCCT; M75.3) represents a specific subgroup

From the Department of Orthopaedic Surgery, Spaarne Gasthuis, Hoofddorp (J.K.G.L., I.N.S., A.v.N.); Coronel Institute of Occupational Health, Amsterdam UMC, University of Amsterdam, Amsterdam Public Health Research Institute, Amsterdam (P.P.F.M.K.); Department of Orthopaedic Surgery, Onze Lieve Vrouwe Gasthuis (OLVG), Amsterdam (M.P.J.v.d.B.); Department of Orthopaedic Surgery, Amsterdam University Medical Center, Amsterdam (B.J.v.R., D.E.); and Department of Orthopaedic Surgery, Amphia, Breda (D.E.), the Netherlands.

The authors report the following potential conflicts of interest or sources of funding: J.K.G.L. reports grants from Spaarne Gasthuis Hospital and nonfinancial support from Richard Wolf GmbH, during the conduct of the study. D.E. reports personal fees from Zimmer Biomet and grants from Stryker, outside the submitted work. A.v.N. reports personal fees from DePuy of shoulder patients with calcific deposits in the tendons. The prevalence of RCCT is between $2.7 \%$ and $10 \%$ in patients without shoulder pain and up to $40 \%$ in symptomatic patients. Clinical symptoms are generally described as activity-related pain similar to subacromial pain syndrome (SAPS). ${ }^{2}$ Klik of tik om tekst in te voeren. The condition mostly affects female patients

Synthes and Link Lima, outside the submitted work. M.P.J.v.d.B. reports grants from Wright Medical Group and Smith $\theta$ Nephew, outside the submitted work. Full ICMJE author disclosure forms are available for this article online, as supplementary material.

Received March 8, 2020; accepted July 21, 2020.

Address correspondence to J. K. G. Louwerens, M.D., Spaarne Gasthuis, Spaarnepoort 1,2134TM.E-mail: jlouwerens@spaarnegasthuis.nl

(C) 2020 THE AUTHORS. Published by Elsevier Inc. on behalf of the Arthroscopy Association of North America. This is an open access article under the CC BY-NC-ND license (http://creativecommons.org/licenses/by-nc-nd/4.0/). 2666-061X/20266

https://doi.org/10.1016/j.asmr.2020.07.021 
(2:1 ratio) who are of working age. ${ }^{3}$ Klik of tik om tekst in te voeren.

The treatment initially consists of physiotherapy, analgesics, and a subacromial infiltration with corticosteroids. ${ }^{4,5}$ When primary treatment fails, minimally invasive therapies like high-energy extracorporeal shockwave therapy (ESWT) and ultrasound-guided needling (UGN) can be considered as an alternative for a surgical intervention. Multiple prospective studies and reviews have analyzed the functional outcome after treatment for patients with RCCT. ${ }^{6,7}$ Klik of tik om tekst in te voeren. However, little is known about the patients' work ability and sick leave before and after minimally invasive treatment for RCCT. Since this condition primarily affects people in their working age, it is of importance to know what the treatment effect is on work ability and sick leave. These are questions that too often remain unanswered in clinical studies. The purposes of this study were to examine the impact of RCCT on patients' self-reported work ability and sick leave, to compare work ability and sick leave with shoulder function after minimally invasive treatment, and to assess which prognostic factors influence the change in work ability. We hypothesized that RCCT would have a significant impact on work ability and sick leave and that this would be correlated with a greater functional disability. With regard to the prognostic factors, we hypothesized that work status (salaried or self-employed), work load, and successful resorption of the deposit would be the most important predictors for change in work ability.

\section{Methods}

The study was designed as a prospective cohort study. The patients were included in a published clinical randomized controlled trial comparing high-energy ESWT with UGN in patients with RCCT with the Constant score as main outcome measure. ${ }^{8}$ Klik of tik om tekst in te voeren. For the current study, both groups were analyzed as a cohort to answer our research questions concerning work ability and sick leave. Patients were included between May 2014 and December 2017. The study was registered in the Dutch clinical trial registration (NL4304/NTR4448) and approved by both the medical ethics review committee (METC, number NL44205.094.13) and the institutional review board (IRB number 2013.26, Spaarne Gasthuis, Hoofddorp, the Netherlands). Informed consent forms were signed by all participating patients.

\section{Study Population}

Sixty-seven consecutive patients referred to the outpatient orthopaedic clinic with clinical signs of nontraumatic anterograde-lateral sided shoulder pain when the arm was elevated were included. Patients were eligible for inclusion whether self-employed or with salaried work. Inclusion criteria for participation in this study were age $>18$ years, clinical sign of SAPS, standardized radiographs showing a calcific deposit in the rotator cuff tendons with a diameter of at least $5 \mathrm{~mm}$ in size, morphologic type I and type II deposits corresponding to the classification of Gärtner and Simons ${ }^{9}$ Klik of tik om tekst in te voeren. (type I, sharply outlined and densely structured; type II, sharply outlined and inhomogeneous or homogenous with no defined border), symptoms for more than 4 months, a completed and unsuccessful nonsurgical treatment program including non-steroidal anti-inflammatory drugs, physiotherapy, and a subacromial infiltration with a corticosteroid. Exclusion criteria were the following: unemployment, ultrasonic signs of a partial or full rotator cuff tendon, clinical or radiographic signs of a resorption phase, calcific deposits in multiple tendons of the rotator cuff, osteoarthritis of the glenohumeral or acromioclavicular joint, adhesive capsulitis, previous shoulder surgery, ESWT or UGN to the affected shoulder, instability of the shoulder, rheumatoid arthritis, neurologic disorders or dysfunction of the upper limb, and the inability to give informed consent.

\section{Inclusion}

The medical history was taken and a clinical examination of the shoulder was performed. Standardized shoulder radiographs (anteroposterior, outlet-, axial-, and acromioclavicular view) and an ultrasound examination of the rotator cuff were obtained. Eligible patients were provided with written and oral information about the trial and had at least 1 week to consider participation. Patients who were willing to participate were contacted by the coordinating investigator (J.L.) for further evaluation and inclusion.

\section{Treatment Procedures}

The ESWT group was treated with 4 sessions of highenergy ESWT with a 1-week interval between sessions. Each session consisted of 2000 piezoelectric pressure pulses at a frequency of $4 \mathrm{~Hz}$ with a total energy flux density of $0.351 \mathrm{~mJ} / \mathrm{mm}^{2}$ resulting in a total energy amount of $2808 \mathrm{~mJ}$. The Piezowave2 system (Richard Wolf GmbH, Knittlingen, Germany) was used as ESWT device. The UGN group was treated with a single procedure, performed in an outpatient clinical setting by 1 orthopaedic shoulder surgeon (A.v.N.) assisted by an experienced ultrasonographer. A double-needle UGN technique with aspiration and lavage of the calcific deposit was used. After the UGN procedure, one of the needles was placed in the subacromial bursa under ultrasound guidance and a mixture of $4 \mathrm{cc}$ bupivacaine $0.5 \%$ (Pfizer Inc, New York, NY) and 1 cc Depo-Medrol $40 \mathrm{mg} / \mathrm{mL}$ (Pfizer Inc) was injected.

After treatment, both groups followed a standardized physical therapy program including active and passive 
Table 1. Demographics and Baseline Characteristics

\begin{tabular}{|c|c|}
\hline & Total $(\mathrm{n}=67)$ \\
\hline Sex, female, n (\%) & $45(67)$ \\
\hline Age, mean (SD) & $49.6(6.4)$ \\
\hline BMI, mean (SD) & $25.3(3.7)$ \\
\hline Non-musculoskeletal comorbidities, n (\%) & $25(37)$ \\
\hline Duration of complaints, y, mean (SD) & $3.2(3)$ \\
\hline Dominant side affected, n (\%) & $43(64)$ \\
\hline Size of calcific deposit, mm, mean (SD) & $15.7(6.2)$ \\
\hline \multicolumn{2}{|l|}{ Location, n (\%) } \\
\hline Supraspinatus & $58(87)$ \\
\hline Infraspinatus & $5(8)$ \\
\hline Subscapularis & $4(6)$ \\
\hline \multicolumn{2}{|l|}{ Work status, n (\%) } \\
\hline Salaried & $52(78)$ \\
\hline Self-employed & $15(22)$ \\
\hline \multicolumn{2}{|l|}{ Physical workload, n (\%) } \\
\hline Light work & $39(58)$ \\
\hline Medium work & $16(24)$ \\
\hline Heavy work & $12(18)$ \\
\hline Working hours a week (mean) & $31.0(10.7)$ \\
\hline \multicolumn{2}{|c|}{ Self-reported work-relatedness of symptoms, n (\%) } \\
\hline Related & $14(21)$ \\
\hline Not related & $17(25)$ \\
\hline Don't know & $36(54)$ \\
\hline CMS, mean (SD) & $66.9(12.1)$ \\
\hline DASH, mean (SD) & $37.4(15.4)$ \\
\hline VAS pain, mean (SD) & $5.9(1.6)$ \\
\hline
\end{tabular}

BMI, body mass index, CMS, Constant-Murley Score, DASH, Disabilities of Arm, Shoulder and Hand score, SD, standard deviation, VAS, visual analog score.

exercise mobilization techniques (centric and eccentric rotator cuff-strengthening exercises in combination with scapular stabilization) to increase power and range of motion and prevent muscular deficit or imbalance. Oral analgesics were administered for a maximum of 7 days postintervention when necessary. The use of additional over-the-counter analgesics was not systematically monitored.

\section{Work Ability and Sick Leave}

At baseline, all patients were asked if they were selfemployed or had a salaried job. Subsequently, to assess the self-reported work ability, the single-item work ability score (WAS) question concerning the "current work ability compared with the lifetime best," with a score of 0 ("completely unable to work") to 10 ("work ability at its lifetime best") was used as the primary outcome of this study. ${ }^{10-12}$ Klik of tik om tekst in te voeren. The designers of the method suggested the following categorization: poor (0-5 points), moderate (6-7), good (8-9), excellent (10). ${ }^{13,14}$ Klik of tik om tekst in te voeren. The WAS is part of the Work Ability Index, a 7-part self-assessment questionnaire. The following aspects of the patients' working situation also were selfreported using the Work Ability Index: number of working hours per week; absenteeism from work in the last month due to shoulder complaints (yes/no; number of days), and whether the patient thought their shoulder complaints were work-related. Secondary work questions were related to the amount of work (quantity), the quality of work, and the experienced limitations during work due to their shoulder disorder. The participants' jobs were classified as light, medium, or heavy, corresponding with the physical demands of their work for the shoulder. These physical demands were based on the evidence-based exposure criteria for the workrelatedness of SAPS by the Dutch Center for Occupational Diseases. ${ }^{15}$ Two occupational health experts independently scored all jobs, and disagreements were resolved by discussion.

Six months and 1 year after treatment, patients were asked to answer the same Work Ability Index. Based on the difference in pre- and post-treatment employment, working hours, and work-ability scores, sick leave was classified as full return to work (no experienced limitations), partial return to work (partial improvement of work-ability and working hours), and full-time sick leave.

\section{Clinical and Radiographic Evaluations}

The following patient characteristics were collected: sex, age (years), body mass index, comorbidities, duration of symptoms, and hand dominance. At baseline and after 6 months and 1 year, the ConstantMurley score, ${ }^{16}$ the Disabilities of the Arm, Shoulder and Hand questionnaire (DASH), ${ }^{17}$ and a visual analog score (VAS), for average pain over a l-week period were used for the clinical assessment. The minimal clinically important difference for the Constant-Murley score (8.3 points), DASH (10.2 points), and VAS pain (2.1 points) were determined based on available literature. ${ }^{18}$ The size, morphology, and amount of resorption (full, more than $50 \%$, less than $50 \%$, no change) of the calcific deposit were analyzed by standard shoulder radiographs at baseline and after 6 months. The length of the deposit was measured in terms of the maximum size of the longest axis in any direction. The radiographs were graded by another physician, who was blinded for the allocated treatment.

\section{Statistical Analysis}

Statistical analyses were performed by using SPSS, version 26.0 (IBM Corp., Armonk, NY). Continuous data are presented as means with standard deviations or $95 \%$ confidence intervals, and categorical variables as frequencies with accompanying proportions. Change of work-related and clinical outcome between follow-up moments was assessed with paired $t$-tests or McNemar tests or Wilcoxon signed rank tests where appropriate. To identify predictive factors for change in WAS, all potential predictive factors (treatment method [ESWT vs UGN], age, sex, resorption [less or more than $50 \%$ ], physical work lead [light vs medium/heavy], and work 
Table 2. Work-Related and Clinical Outcome

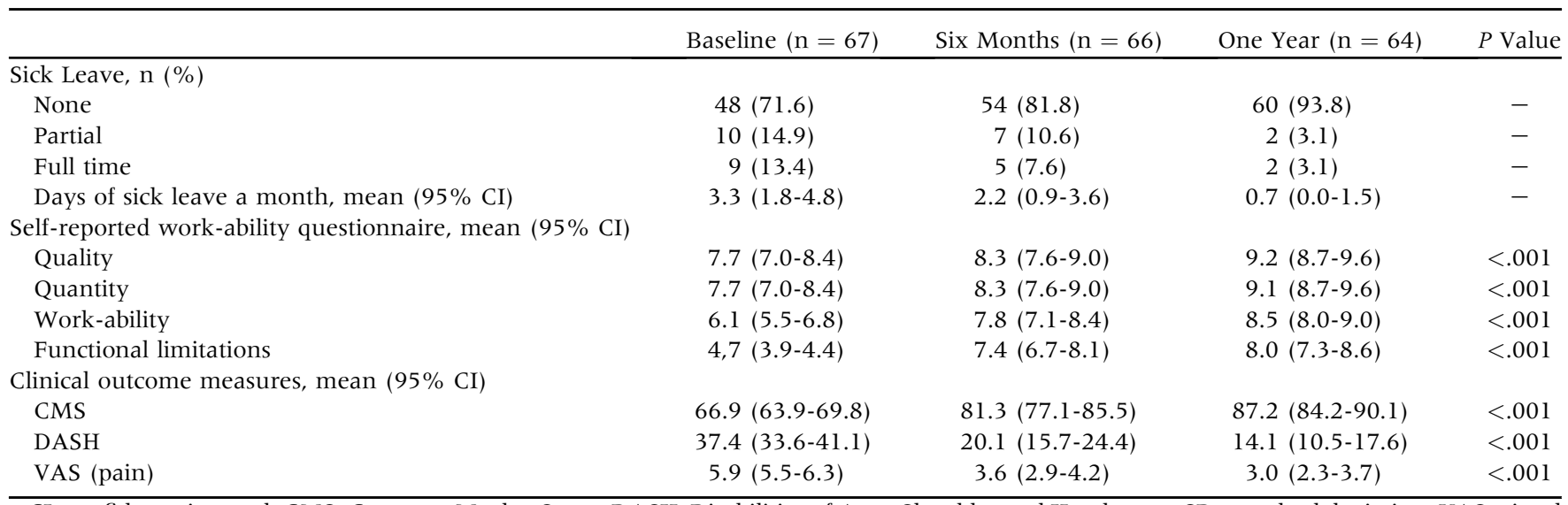

CI, confidence interval; CMS, Constant-Murley Score; DASH, Disabilities of Arm, Shoulder and Hand score; SD, standard deviation; VAS, visual analog score.

status [salaried vs self-employed]) were initially tested by univariate linear regression analyses. In case of significant association (adjusted significance level of 0.10), these factors were entered in a multivariate regression model.

\section{Results}

\section{Baseline Characteristics}

Between May 2014 and December 2017, a total of 67 patients were included (Table 1). One patient was lost to follow-up after 6 months. The mean age was $49.6 \pm$ 6.4 years and $45(67 \%)$ were female. The mean duration of symptoms was $3.2 \pm 3.0$ years, and the supraspinatus was the most frequently $(87 \%)$ affected tendon. Most patients were employees (78\%). Physical workload was categorized as light $(58 \%)$, medium $(24 \%)$, and heavy $(18 \%)$. Nineteen patients reported sick leave $(28 \%)$, of whom 9 patients $(13 \%)$ were on permanent sick leave before treatment. Twenty-one percent of the patients stated that their shoulder symptoms were work-related.

\section{Work-Related and Clinical Outcomes}

Table 2 presents the work-related outcomes of this study. Between 6 months and 1 year, 2 patients lost their job because of nonshoulder-related reasons. WAS improved from a mean score of $6.1 \pm 2.8$ to $8.5 \pm 2.0$ after 1 year. The change from baseline scores for workrelated factors improved significantly for all 4 subcategories: work ability, quality of work, quantity of work, and functional limitations. Of the 9 patients who reported to be on full-time sick leave, 5 patients were still on full-time sick leave after 6 months and 2 after 1 -year follow-up. The percentage of patients reporting sick leave was reduced from 19 patients $(28 \%)$ to 4 patients $(6 \%)$, with a decline in sick leave from a mean of 3.3 days a month to 0.7 days a month.

Overall, the Constant-Murley score, DASH, and VAS pain scores at final follow-up improved with clinically relevant differences in comparison with the baseline scores (Table 2). Radiographic resorption was complete in $53 \%$, almost complete in $18 \%$, minimally changed in $9 \%$, and unchanged in $20 \%$. Patients who were with partial- or full-time sick leave at baseline had

Table 3. Prognostic Variables for Work Ability

\begin{tabular}{|c|c|c|c|c|}
\hline & \multicolumn{2}{|c|}{ Six Months } & \multicolumn{2}{|c|}{ One Year } \\
\hline \multicolumn{5}{|l|}{ Univariate } \\
\hline Sex & $0.43(-1.02 ; 1.89)$ & .56 & $0.53(-0.79 ; 1.84)$ & .43 \\
\hline High-energy ESWT vs UGN & $0.09(-1.28 ; 1.47)$ & .90 & $0.74(-0.50 ; 1.99)$ & .24 \\
\hline Resorption of the calcific deposit & $0.64(-0.87 ; 2.15)$ & .40 & $1.08(-0.27 ; 2.43)$ & .11 \\
\hline Workload (light vs medium/heavy) & $1.43(0.08 ; 2.79)$ & .04 & $1.55(0.33 ; 2.76)$ & .01 \\
\hline \multicolumn{5}{|l|}{ Final model } \\
\hline Workload (light vs medium/heavy) & $1.43(0.08 ; 2.79)$ & .04 & $1.55(0.33 ; 2.76)$ & .01 \\
\hline
\end{tabular}

NOTE. Boldface indicates statistical significance.

ESWT, extracorporeal shockwave therapy; UGN, ultrasound-guided needling. 
Fig 1. Difference in change from baseline work ability scores after 6 months and 1 year between the 3 physical workload categories. ${ }^{*} P<.001$ in comparison with the category "low."

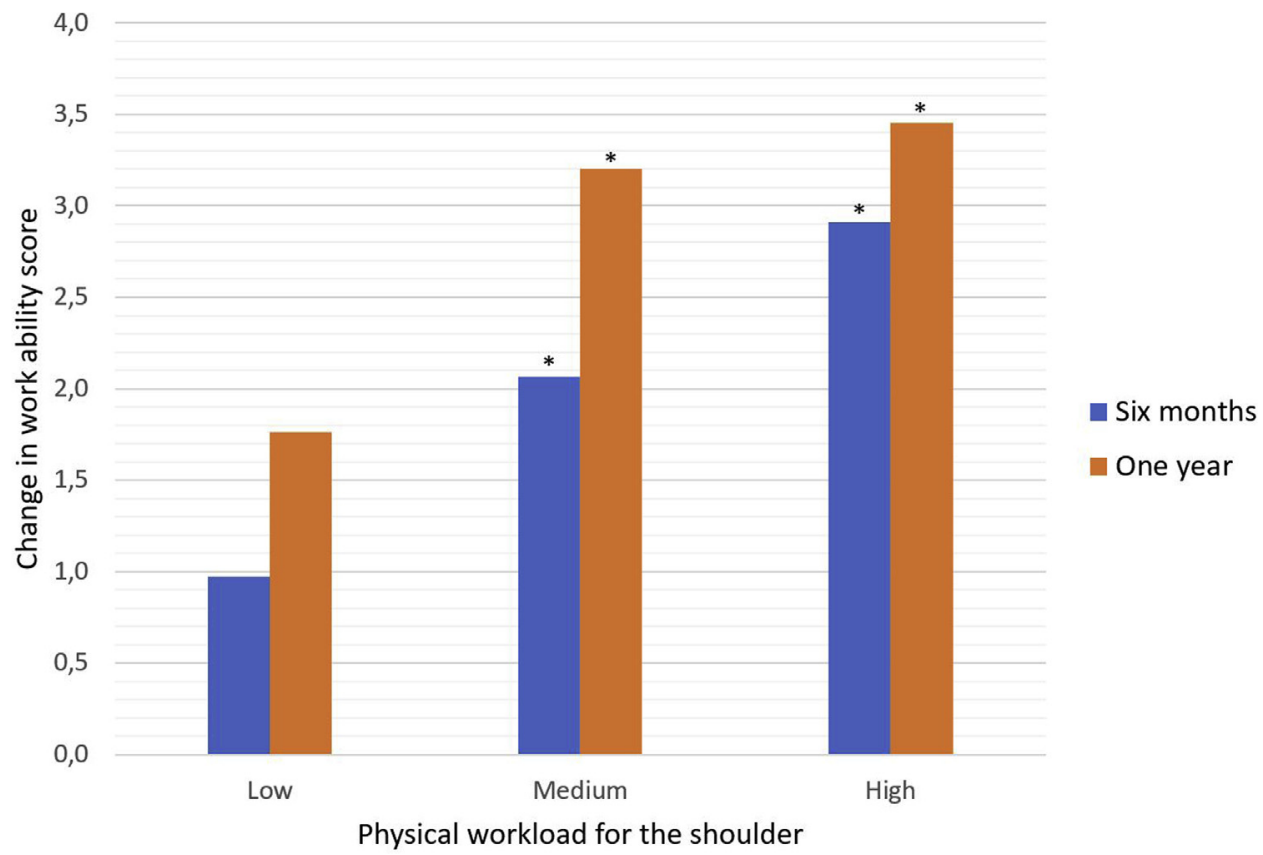

significantly lower Constant-Murley scores (58.5 \pm 9.4 vs $70.2 \pm 11.4 P \leq .001)$ and DASH scores $(48.5 \pm 15.3$ vs $32.9 \pm 13.2 P \leq .001)$ when compared with the group without sick leave. After 6 months $(P=.05)$ and 1 year $(P=.006)$, this difference remained significant for the DASH score.

\section{Prognostic Variables for the WAS}

Table 3 presents the results of the univariate analyses of each potentially prognostic factor for the change in WAS, as well as the final model. After 6-month and 1-year follow-up, only the physical work load, defined as light versus medium and heavy combined, was a predictive factor for a significant change in WAS $(P=.01)$ with a $\beta$-coefficient of $1.43(95 \%$ confidence interval 0.08-2.79) in favor of the high-workload group. All other factors were not significantly associated with the change in WAS. Fig 1 graphically shows that the change from baseline scores for WAS in the light-workload group (1.0 after 6 months and 1.8 after 1 year) was significantly lower than the scores in the medium physical workload group (2.1 after 6 months and 3.2 after 1 year) and high physical workload group (2.9 after 6 months and 3.5 after 1 year).

\section{Discussion}

The principal finding of this study is that RCCT has a significant impact on patients' work ability and sick leave. Treatment with minimally invasive treatment techniques was associated with a reduction in partial or full-time sick leave from $28 \%$ to $6 \%$. The mean days of sick leave a month declined from 3.3 to 0.8 days. WAS improved from a mean score of $6.1 \pm 2.8$ to $8.5 \pm 2.0$ after 1 year. The physical workload turned out to be the most important patient-associated factor predicting change in WAS. In particular, the patients with medium-to-high physically demanding work improved the most. This is important information for the clinicians when discussing treatment options with their patients, and it might encourage patients with high physical demanding work to choose minimally invasive therapies.

Not much is known about the impact of RCCT on work ability and sick leave. Since this condition primarily affects patients of working age, information regarding these outcome measures is of great importance for both clinicians and patients. However, none of the 16 randomized controlled clinical trials analyzed in a previous meta-analysis ${ }^{77}$ discussed work-related outcome measures. Although data on RCCT are scarce, multiple studies have been published on workrelated risk factors for SAPS. ${ }^{15,19-21}$ For work-related specific shoulder disorders, the biomechanical factor seems to be the most important, but psychosocial factors might also contribute. Van Rijn et al. ${ }^{19}$ concluded in their systematic review that forceful exertion in work, highly repetitive work, awkward postures, and high psychosocial job demand are associated with the occurrence of shoulder disordersKlik of tik om tekst in te voeren. This was confirmed by a more recent metaanalysis by van der Molen et al. ${ }^{15}$ stating that there is moderate GRADE level evidence for a 2-fold chance of developing SAPS when being exposed to arm elevation and shoulder load during work. ${ }^{15}$ 
In this study, the physical demands were classified based on the evidence-based exposure criteria for the work-relatedness of SAPS by the Dutch Center for Occupational Diseases. We found that a greater percentage of patients in medium and high physical demand jobs were on (partial) sick leave at baseline and reported a significant lower WAS with low functional performance scores. The present data support the hypothesis that in particular patients with medium or high physically demanding work for the shoulder might benefit from minimally invasive treatment.

The other factors that were tested in our predictive model were not significantly associated with the change in WAS. Engebretsen et al. $^{20}$ found, in their RCT comparing radial ESWT and supervised exercise in patients with SAPS, that 12 or fewer years of education is the most consistent predictive factor for absenteeism and low functional outcome scores after 1 year. While they did not report the workload of these patients, there is probably a strong relationship between a lower education level and performance of higher physically demanding work. Seil et al. ${ }^{22}$ reported no difference in return to work between difference physical work load categories in a retrospective cohort study analyzing surgical outcomes for RCCTKlik of tik om tekst in te voeren. They concluded that the only difference in time to return to work was due to the presence of disability claims.

A strength of this study is that it contains 1-year followup data on both validated work-related outcome measures and clinical outcome measures. Moreover, the patients worked in a wide variety of occupational settings, which makes the results more generalizable than a selective sample of workers. Although the numbers in this study were relatively small, data from $95 \%$ of the patients were available at 1-year follow-up and differences statistically tested.

Work-related outcome measures should be included more frequently in orthopaedic surgery research, as these parameters are relevant in the treatment of working-age patients and are frequently not reported in clinical trials. The use of a single-item measure has been validated in previous studies and has numerous advantages: it is short in length, requires less time to complete, and is more likely to be completed by the employee in comparison with multiple-item questionnaires. ${ }^{23-25} \mathrm{Klik}$ of tik om tekst in te voeren. Furthermore, it has been shown not to decrease the validity of the work ability information collected in comparison with multiple-item questionnaires.

Self-reported variables such as self-reported likelihood of the work-relatedness of the musculoskeletal disease, rating of the expected effectiveness of work-related interventions, presence of support from supervisors, and presence of modifiable job duties can play an important role in the assessment of work-related outcome measures. Furthermore, it would be interesting to validate if patient with high physical demanding jobs for the shoulder benefit more from minimal invasive treatment options than patients with low physical demanding jobs.

\section{Limitations}

One of the limitations of this study is that it did not include a control group and therefore we could not compare the outcome with patients receiving no treatment. Furthermore, since the study was primarily powered to look for a difference in the clinical Constant-Murley scores, it might have been underpowered for the outcome measure work ability and sick leave. Finally, since this study focused specifically on RCCT, statements made in this study are not generalizable to other conditions that cause SAPS.

\section{Conclusions}

This study supports the hypothesis that rotator cuff calcific tendinitis has a significant impact on work ability and sick leave. Minimally invasive treatment resulted in a clinically relevant improvement in work ability score and decline in sick leave. In particular, patients with medium and high physically demanding work for the shoulder benefit from minimally invasive treatment to improve their work ability.

\section{Acknowledgments}

The authors acknowledge all the colleagues of the Department of Orthopaedic Surgery, Spaarne Gasthuis, the Netherlands, for their help in the logistics, inclusion, treatment, and planning of the patients with a commendation for Nienke Miedema (nurse practitioner), Jeannette Verhart (research nurse), Marjolein Schager (research nurse), Tjarco Alta (MD), Nick Leegwater (MD), Christiaan van Bergen (MD), Erik Versluis (PT), Erik Kramer (PT), and Rob Boonstra (PT).

\section{References}

1. Greving K, Dorrestijn O, Winters JC, et al. Incidence, prevalence, and consultation rates of shoulder complaints in general practice. Scand J Rheumatol 2012;41:150-155.

2. Louwerens JKG, Sierevelt IN, van Hove RP, van den Bekerom MPJ, van Noort A. Prevalence of calcific deposits within the rotator cuff tendons in adults with and without subacromial pain syndrome: Clinical and radiologic analysis of 1219 patients. J Shoulder Elbow Surg 2015;24: 1588-1593.

3. Oliva F, Via AG, Maffulli N. Physiopathology of intratendinous calcific deposition. BMC Med 2012;10:95.

4. Ogon P, Suedkamp NP, Jaeger M, Izadpanah K, Koestler W, Maier D. Prognostic factors in nonoperative therapy for chronic symptomatic calcific tendinitis of the shoulder. Arthritis Rheum 2009;60:2978-2984.

5. Suzuki K, Potts A, Anakwenze O, Singh A. Calcific tendinitis of the rotator cuff: management options. J Am Acad Orthop Surg 2014;22:707-717. 
6. Lafrance S, Doiron-Cadrin P, Saulnier M, et al. Is ultrasound-guided lavage an effective intervention for rotator cuff calcific tendinopathy? A systematic review with a meta-analysis of randomised controlled trials. BMJ Open Sport Exerc Med 2019;5.

7. Louwerens JKG, Sierevelt IN, van Noort A, van den Bekerom MPJ. Evidence for minimally invasive therapies in the management of chronic calcific tendinopathy of the rotator cuff: A systematic review and meta-analysis. J Shoulder Elbow Surg 2014;23:1240-1249.

8. Louwerens J, Sierevelt IN, Kramer ET, et al. Comparing ultrasound-guided needling combined with a subacromial corticosteroid injection versus high-energy extracorporeal shockwave therapy for calcific tendinitis of the rotator cuff. A randomized controlled trial. Arthroscopy 2020;36: 1823-1833.

9. Gärtner J, Simons B. Analysis of calcific deposits in calcifying tendinitis. Clin Orthop Relat Res 1990;254: $111-120$.

10. de Zwart BCH, Frings-Dresen MHW, van Duivenbooden JC. Test-retest reliability of the Work Ability Index questionnaire. Occup Med 2002;52:177-181.

1 1. Tuomi K, Ilmarinen J, Jahkola A, Katajarinne L, Tulkki A. Work ability index, 2nd ed. Helsinki: Finnish Institute of Occupational Health, 1998.

12. Kuijer PPFM, Gouttebarge V, Wind $H$, van Duivenbooden C, Sluiter JK, Frings-Dresen MHW. Prognostic value of self-reported work ability and performance-based lifting tests for sustainable return to work among construction workers. Scand J Work Environ Health 2012;38:600-603.

13. Gould R, Ilmarinen J, Järvisalo J. Dimensions of work ability. Results from the Health 2000 survey. Occup Environ Health 2008;81:495-501.

14. Kujala V, Tammelin T, Remes J, Vammavaara E, Ek E, Laitinen J. Work ability index of young employees and their sickness absence during the following year. Scand $J$ Work Environ Health 2006;32:75-84.

15. van der Molen HF, Foresti C, Daams JG, FringsDresen MHW, Kuijer PPFM. Work-related risk factors for specific shoulder disorders: A systematic review and metaanalysis. Occup Environ Med 2017;74:745-755.

16. Constant CR, Murley AH. A clinical method of functional assessment of the shoulder. Clin Orthop Relat Res 1987;214: 160-164.

17. Dixon D, Johnston M, McQueen M, Court-Brown C. The Disabilities of the Arm, Shoulder and Hand Questionnaire (DASH) can measure the impairment, activity limitations and participation restriction constructs from the International Classification of Functioning, Disability and Health (ICF). BMC Musculoskelet Disord 2008;9:114.

18. Hao Q, Devji T, Zeraatkar D, et al. Minimal important differences for improvement in shoulder condition patient-reported outcomes: A systematic review to inform a BMJ Rapid Recommendation. BMJ Open 2019;9:1-10.

19. van Rijn RM, Huisstede BM, Koes BW, Burdorf A. Associations between work-related factors and specific disorders of the shoulder-A systematic review of the literature. Scand J Work Environ Health 2010;36:189-201.

20. Engebretsen K, Grotle M, Bautz-Holter E, Ekeberg OM, Brox JI. Predictors of Shoulder Pain and Disability Index (SPADI) and work status after 1 year in patients with subacromial shoulder pain. BMC Musculoskelet Disord 2010;11:218.

21. Miranda H, Viikari-Juntura E, Martikainen R, Takala EP, Riihimäki H. A prospective study of work related factors and physical exercise as predictors of shoulder pain. Occup Environ Med 2001;58:528-534.

22. Seil R, Litzenburger H, Kohn D, Rupp S. Arthroscopic treatment of chronically painful calcifying tendinitis of the supraspinatus tendon. Arthroscopy 2006;22:521-527.

23. Nagy MS. Using a single-item approach to measure facet job satisfaction. J Occup Organ Psych 2002;75:77-86.

24. Wanous JP, Reichers AE, Hudy MJ. Overall job satisfaction: How good are single-item measures? J Appl Psychol $1997 ; 82: 247-252$.

25. el Fassi M, Bocquet V, Majery N, Lair ML, Couffignal S, Mairiaux P. Work ability assessment in a worker population: comparison and determinants of Work Ability Index and Work Ability score. BMC Public Health 2013;13:305. 